\title{
Expression of intracellular adhesion molecule-1 linking superoxide to mobilization of granulocytes and macrophages after periarterial blood in rat femoral artery: effect of rebamipide
}

\author{
Jae Moon Choi, M.D., Chi Dae Kim, M.D., Ph.D., And Ki Whan Hong, M.D., Ph.D. \\ Department of Pharmacology, College of Medicine, Pusan National University, Pusan, Korea
}

\begin{abstract}
Object. To clarify the mechanism(s) involved in the perivascular mobilization of granulocytes and macrophages by periarterial autologous blood (PAAB) in the vicinity of the femoral artery (FA) in rats, superoxide production as well as expression of intercellular adhesion molecule-1 (ICAM-1) were determined by conducting both in vitro and in vivo experiments.

Methods. In an in vitro study, a significant amount of superoxide inhibited by diphenyleneiodonium $(20 \mu \mathrm{M}$ and $100 \mu \mathrm{M}$ ) was identified at 3 hours after application of $10 \%$ whole blood to the aortic segments, and these results were correlated with in vitro ICAM-1 expression. High expression of ICAM-1 was subsequently demonstrated in these segments at 24 hours in in vitro and in vivo studies. In the in vivo study, an increased mobilization of granulocytes paralleled with a high expression of ICAM-1 in the vessels at 24 hours after administration of PAAB to the FA and then declined. Subsequently, macrophage infiltration progressively increased at all layers throughout a period of 7 to 12 days. Pretreatment with rebamipide (100 and $300 \mathrm{mg} \mathrm{kg}^{-1}$ day $^{-1}$, orally) significantly inhibited the expression of ICAM1 with inhibition of mobilization of granulocyte/macrophage.

Conclusions. These findings suggest that application of PAAB to the rat FA causes superoxide-linked expression of ICAM-1 and mobilization of granulocyte and macrophages. Thus, the potential value in suppressing these variables stimulated by PAAB is indicated in therapeutic strategies for prevention and possible regression of vasospasm after subarachnoid hemorrhage.
\end{abstract}

KeY WoRDS - superoxide dismutase subarachnoid hemorrhage granulocyte • macrophage • vasospasm •
Evidence reported in several studies indicates the importance of hemoglobin in the pathogenesis of vasospasm after SAH. ${ }^{4,16,32}$ A vasospasm model of rat FA in which whole blood was applied to the adventitial surface of the FA has been reported by Okada, et al., ${ }^{18}$ They demonstrated a chronic narrowing of the FA after the application of periadventitial blood, which was analogous in degree and in time course to that observed in cerebral vasospasm after SAH. This arteriopathy appears to be closely related with immunological and inflammatory responses based on several lines of evidence - that is, the presence of inflammatory cells $s^{5,24}$ increased levels of immunoglobulins and complements in the serum and vessel walls, ${ }^{23}$ and inhibition of the vasospasm by the antiinflammatory drugs and immunosuppressants. ${ }^{10,21}$ An expression of ICAM-1 was demonstrated at the intimal and medial layers of the basilar artery of SAH-induced rats ${ }^{6}$ and in the FA subjected to

Abbreviations used in this paper: $\mathrm{CL}=$ chemiluminescence; DDTC = diethyldithiocarbomate; DPI = diphenyleneiodonium; FA $=$ femoral artery; $\mathrm{SAH}=$ subarachnoid hemorrhage; $\mathrm{SOD}=$ superoxide dismutase. application of PAAB. ${ }^{28}$ Hemoglobin was demonstrated to generate the superoxide anion by autooxidation of hemoglobin to methemoglobin. ${ }^{17}$ With these results, a growing body of evidence has accumulated concerning the possible role of free radical reactions in the pathogenesis of prolonged cerebral vasospasm following SAH from ruptured intracranial aneurysms. ${ }^{1,25}$

In our previous experiments we have documented the pharmacological actions of rebamipide, 2-(4-chlorobenzoylamino)-3-[2-(1H)-quinolinon-4-yl]propionic acid, in that it prevented ischemia/reperfusion-induced gastric mucosal damage through inhibition of mobilization of neutrophils via inhibition of production of reactive $\mathrm{O}_{2}$ species from activated neutrophils. ${ }^{11}$ Furthermore, it was shown to suppress the adherence of neutrophils to endothelial cells by inhibiting the expression of CD11b on neutrophils and interleukin- 8 expression on the gastric epithelial cell. ${ }^{13}$ The evidence that adhesion-dependent inflammatory response is primarily mediated by expression of CD11b/CD18 on neutrophils and ICAM-1 on endothelial cells ${ }^{14}$ leads to the prediction that rebamipide may potentially inhibit the ICAM-1-coupled mobilization of granulocytes/macrophages. 
To identify the superoxide anion-linked ICAM-1 expression, we measured superoxide anion production from an aortic segment on exposure to whole blood by lucigenin CL and expression of ICAM-1 by immunohistochemistry (in vitro). After application of autologous blood in the vicinity of rat FA (in vivo), we identified the SOD anion-linked ICAM-1 expression and mobilization of granulocyte/macrophage in the FA, and we further examined these variables after pretreatment with rebamipide.

\section{MATERIALS AND METHODS}

\section{Application of Whole Blood to the Femoral Artery}

As an in vivo experiment, male Splague-Dawley rats weighing 270 to $330 \mathrm{~g}$ were anesthetized with an intraperitoneal injection of chloral hydrate $(400 \mathrm{mg} / \mathrm{kg})$ and allowed to breathe spontaneously. Using sterile technique, a 1-cm-long section of FA was exposed in the inguinal region. Autologous whole blood $(0.1 \mathrm{ml})$ obtained from the adjacent femoral vein was infused between silicone tubing and the right FA, and allowed to clot for a few minutes. Thereafter, both ends of tubing were lightly tied with surgical thread to prevent the tubing from falling out from the artery. As a control, the left FA was prepared similarly but with normal saline rather than whole blood. After exposure for a specified time, both FAs were isolated and frozen with liquid nitrogen and stored at $-70^{\circ} \mathrm{C}$ until sectioning. Rebamipide (30, 100, and $300 \mathrm{mg} \mathrm{kg}^{-1}$ day $^{-1}$ ) was administered orally for 3 days before and during application of PAAB.

As an in vitro experiment, isolated segments of rat FA were prepared in the cold buffer $\mathrm{A}$ with the following composition (in $\mathrm{mM}$ ): $\mathrm{NaCl} 118.3, \mathrm{KCl} 4.7, \mathrm{MgSO}_{4} 0.6$, $\mathrm{KH}_{2} \mathrm{PO}_{4} 1.2, \mathrm{NaHCO}_{3} 25, \mathrm{CaCl}_{2} 2.5$, ethylenediaminetetraacetic acid 0.026 , glucose 5.5; $\mathrm{pH} 7.4$, saturated with $95 \% \mathrm{O}_{2}-5 \% \mathrm{CO}_{2}$ mixture and placed at $37^{\circ} \mathrm{C}$ for a $30-$ minute equilibration. The segments were transferred to the DMEM containing 10\% fetal calf serum, $100 \mu \mathrm{M}$ DDTC (an endogenous SOD inhibitor), $100 \mu \mathrm{M}$ NADH and 100 $\mu \mathrm{M}$ NADPH in the absence and presence of $10 \%$ of the autologous whole blood and placed in the $\mathrm{CO}_{2}$ incubator for 24 hours. The segments were then frozen in optimal cooling temperature compound with liquid nitrogen and stored at $-70^{\circ} \mathrm{C}$ until sectioned.

\section{Measurement of Superoxide Anion in Rat Aorta After Application of Blood}

Isolated segments of rat thoracic aorta were placed in the buffer A saturated with the $95 \% \mathrm{O}_{2}-5 \% \mathrm{CO}_{2}$ mixture at $37^{\circ} \mathrm{C}$ for a 30 -minute equilibration. The 3 -mm-long segments were placed in DMEM containing $100 \mu \mathrm{M}$ DDTC in the absence and presence of $10 \%$ of the autologous whole blood and placed in the $\mathrm{CO}_{2}$ incubator for 3 hours. Antagonists were included in the medium during incubation. Thereafter, the segments were removed to 200 $\mu \mathrm{l}$ Krebs-HEPES buffer with a composition (in $\mathrm{mM}$ ): $\mathrm{NaCl} 118.3, \mathrm{KCl} 4.69, \mathrm{MgSO}_{4} 1.2, \mathrm{~K}_{2} \mathrm{HPO}_{4} 1.03$, $\mathrm{NaHCO}_{3} 25$, HEPES 20.0, $\mathrm{CaCl}_{2} 1.87$, glucose 5.5; pH 7.4 , in a 96 -well plate containing lucigenin $(250 \mu \mathrm{M})$ with NADPH and NADH (100 $\mu \mathrm{M}$ each). The plate was then put in a microplate luminometer. The luminometer was set to report arbitrary units of light emitted and integrated over a 40-second interval. Repeated measurements were obtained during 15 minutes and were then averaged. The vessel segments were dried at $80^{\circ} \mathrm{C}$ for 24 hours and the weight measured.

\section{Immunohistochemistry for ICAM-1, Granulocytes, and Macrophages}

The FA was sectioned in a coronal plane by using a cryostat at $-20^{\circ} \mathrm{C}$ with a thickness of $5 \mu \mathrm{m}$. Sectioned preparations were air-dried overnight, fixed in cold acetone and hydrated in PBS. Endogenous tissue peroxidase activity was inhibited by immersing the specimens in the $0.6 \%$ hydrogen peroxide in PBS solution. After washing in PBS, preparations were incubated with $10 \%$ normal horse serum for 30 minutes at $37^{\circ} \mathrm{C}$ to block the nonspecific binding activity. The preparations were incubated in primary antibody at $4^{\circ} \mathrm{C}$ overnight. The mouse anti-rat CD54 (ICAM-1), HIS48 (granulocyte), and ED1 (macrophage) monoclonal antibodies were diluted 1:500, 1:50, and 1:1000 in PBS with 3\% normal horse serum, respectively. Negative controls were incubated with an equivalent volume of diluent solution alone. After primary incubation, the sections were washed three times in PBS and incubated for 1 hour at room temperature with the biotinylated antimouse secondary antibody, adsorbed with rat serum (1:100). After the slides were washed three times with PBS, they were incubated with avidin-biotin horseradish peroxidase macromolecular complex for 1 hour. The slides were washed in PBS and incubated with diaminobenzidine for a specified duration. The reactions were quenched by placement in running tap water, counterstained with hematoxylin, and then the slides were dehydrated in alcohol, cleaned in xylene, and mounted. The expression of ICAM-1 was evaluated with summation of the positively stained area. The mobilization of granulocytes and macrophages was determined by counting the positively stained cells on the vessel wall and blood clot by using an image analyzer. For each animal, at least three sections of the FA were examined.

\section{Pharmacological Agents}

Rebamipide was dissolved in $10 \mathrm{mM} \mathrm{NaOH}$ solution at a concentration of $60 \mathrm{mM}$ for in vitro experiment and suspended in $0.5 \%$ methylcellulose for oral administration. We dissolved DPI in dimethyl sulfoxide at a concentration of $50 \mathrm{mM}$.

\section{Statistical Analysis}

The results are presented as mean \pm standard error of the mean. Multiple comparisons were performed using analysis of variance, and statistical differences between groups were determined by Student's t-test. Differences were considered to be significant at $\mathrm{p}<0.05$.

\section{SOURCES OF SUPPLIES AND EQUIPMENT}

The microplate luminometer (MicroLumat LB96P) was purchased from EG\&G (Berthold, Bad Wildbad, Germany). We obtained the mouse anti-rat antibodies from Serotec (Oxford, UK). The rat serum-absorbed anitmouse secondary antibody was obtained from Vector Labor- 


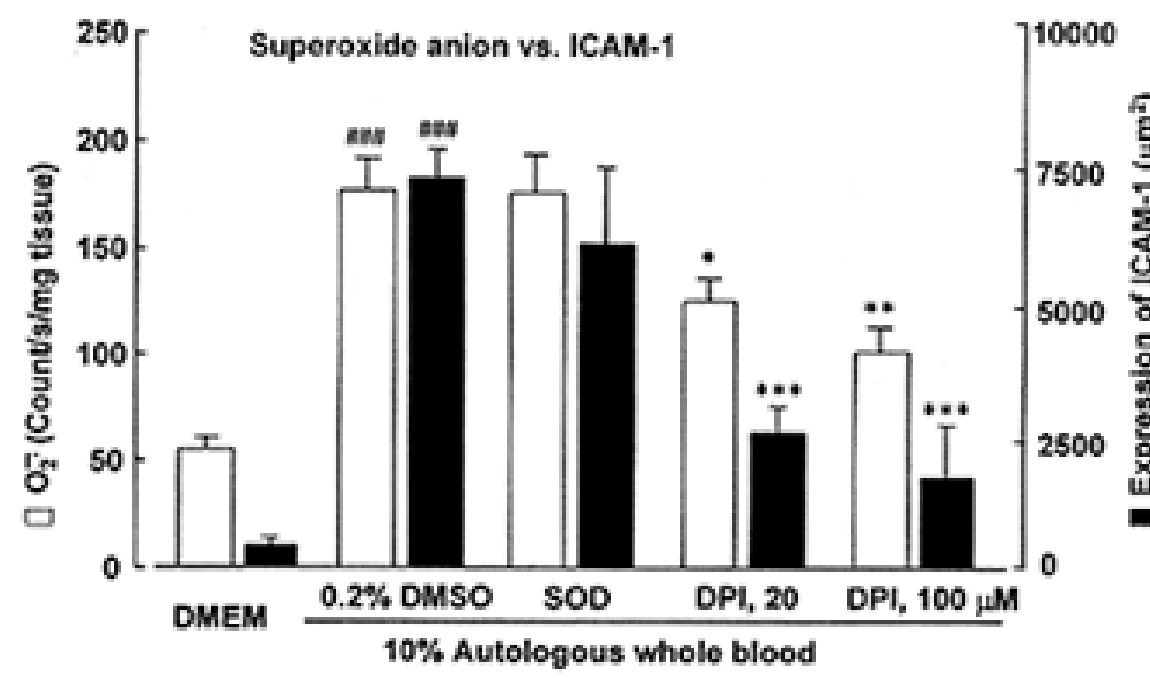

Fig. 1. Bar graph showing in vitro superoxide anion generation and ICAM-1 expression following incubation of rat aortic segments in DMEM containing 10\% autologous whole blood in the absence and the presence of inhibitors. DMSOdimethylsulfoxide; $100 \mathrm{U} / \mathrm{ml}$ superoxide dismutase. Results are expressed as the mean \pm standard error of the mean derived from 4 to 6 experiments. \#\#\# $<0.001$ as compared with DMEM; *p $<0.05$; **p $<0.01$; ***p $<0.001$ as compared with $0.2 \%$ DMSO. $0_{2}^{-}=$Superoxide

atories, Inc. (Burlingame, CA), as were the avidin-biotin horseradish peroxidase complex (Vectastain Elite $\mathrm{ABC}$ kit) and the diaminobenzidine (diaminobenzidine substrate kit). Media Cybernetics, Silver Springs, MD) manufactures the image analyzer (Image-Pro Plus). The rebamipide was donated by Otsuka Pharmaceuticals, Co., Ltd. (Tokushima, Japan). The DPI and DDTC were acquired from Sigma Chemical Co. (St. Louis, MO).

\section{RESULTS}

\section{Superoxide Anion Production and ICAM-1 Expression in the in vitro Experiment}

The level of superoxide anion production was measured using luminometer and the CL method. In the aortic segment that was incubated in DMEM for 3 hours, the amount of CL was $56.4 \pm 5.8 \mathrm{count} / \mathrm{sec} / \mathrm{mg}$ tissue weight (12 sections). Incubation of the aortic segment in the DMEM containing of $10 \%$ whole blood caused a prominent increase in lucigenin CL by $183.7 \pm 15.5$ count/ $\mathrm{sec} / \mathrm{mg}$ tissue weight (19 sections). The increment of CL was not affected by the exogenous SOD $(100 \mathrm{U} / \mathrm{ml})$ but was significantly decreased by DPI $(20 \mu \mathrm{M}$ and $100 \mu \mathrm{M})$. The increase in CL was not affected by addition of $0.2 \%$ dimethyl sulfoxide $(177.7 \pm 22.1$ count $/ \mathrm{sec} / \mathrm{mg}$ tissue weight; 8 sections). In in vitro immunohistochemical study with anti-ICAM-1 antibody significant increases in ICAM-1 expression were revealed in the intima and media, which was also suppressed by DPI $(20 \mu \mathrm{M}$ and $100 \mu$ M) (Fig. 1). Similarly, both increased generation of superoxide anion and ICAM-1 expression were inhibited by rebamipide $(30 \mu \mathrm{M}, 100 \mu \mathrm{M}$, and $300 \mu \mathrm{M})$ in a dosedependent fashion (Fig. 2). Therefore, ICAM-1 expression was well correlated with the generation of superoxide anion $\left(r^{2}=0.95, p<0.002\right.$; Fig. 3$)$.

\section{Expression of ICAM-1 and Mobilization of Granulocytes and Macrophages}

Immunohistochemical study with anti-ICAM-1 antibody demonstrated significant increases in ICAM-1 expression in the intima and media of the FA at 24 hours after application of PAAB (see Fig. 4B) and then it returned to the baseline value at 2 days postoperation and thereafter (Fig. 5A). After treatment with rebamipide (100 and $300 \mathrm{mg} \mathrm{kg}^{-1}$ day $^{-1}$ ), the expression of ICAM-1, which was largely increased by PAAB was significantly decreased (Fig. 4C). Baseline expression in the control group (Fig. 4A) was marginally decreased by rebamipide treatment $(\mathrm{p}>0.05)$ These results are summarized in Table 1.

Following application of PAAB, immunohistochemical study with anti-HIS48 antibody revealed a significantly increased infiltration of granulocytes in the region of media and adventitia of the FA by 12 hours ( $p<0.01)$, and the level peaked at 24 hours $(\mathrm{p}<0.001)$ (Fig. 4E), after which it slowly declined to the baseline level (Fig. 5B). Treatment with rebamipide (100 and $300 \mathrm{mg} \mathrm{kg}^{-1}$ day $\left.^{-1}\right)$ significantly inhibited the increased infiltration of granulocytes in the PAAB-treated vessels $(\mathrm{p}<0.01)$ (Table 1 and Fig. 4F).

The macrophage infiltration was identified with antiED1 monoclonal antibody (Fig. 5C). Immunohistochemical staining for macrophages demonstrated little mobilization of macrophages for up to 3 days posttreatment. Thereafter, ED1-positive macrophages were largely recruited to the vessel wall and the blood clot at 7 days (Fig. $4 \mathrm{H})$ and 12 days after application of blood $(\mathrm{p}<0.05$ and $\mathrm{p}<0.01$, respectively). Rebamipide $\left(300 \mathrm{mg} \mathrm{kg}^{-1}\right.$ day $\left.^{-1}\right)$ significantly inhibited the increased PAAB-induced mobilization of macrophage $(p<0.05)$ (Table 1 and Fig. 4I). 
J. M. Choi, et al.

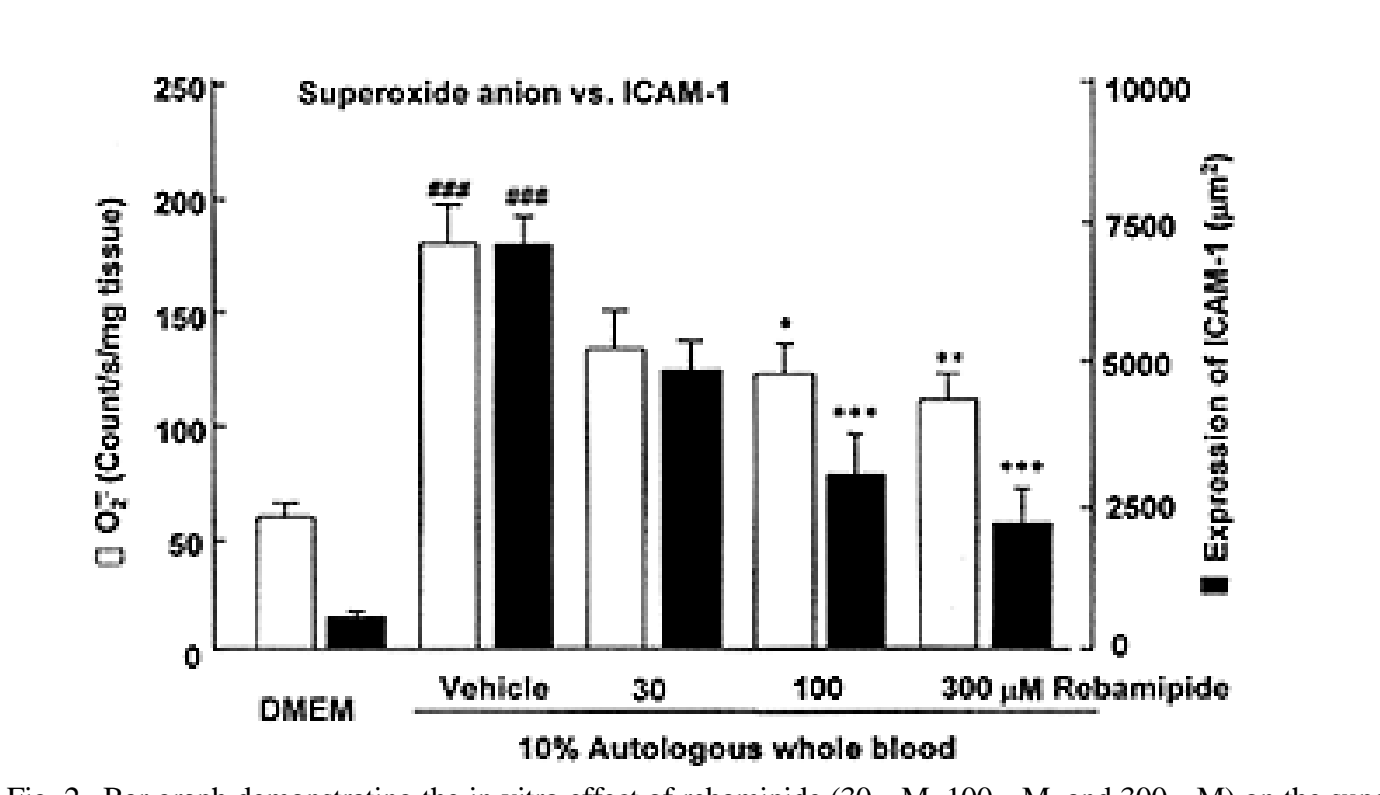

Fig. 2. Bar graph demonstrating the in vitro effect of rebamipide $(30 \mu \mathrm{M}, 100 \mu \mathrm{M}$, and $300 \mu \mathrm{M})$ on the superoxide anion generation and ICAM-1 expression following incubation of rat aortic segments in DMEM containing $10 \%$ autologous whole blood. Results are expressed as mean \pm standard error of the mean derived from four to six experiments. $\# \#$ \# $<0.001$ as compared with DMEM; ${ }^{*} \mathrm{p}<0.05 ; * \mathrm{*} p<0.01 ; * * * \mathrm{p}<0.001$ as compared with vehicle.

\section{DISCUSSION}

The results of the present study clearly demonstrate that application of PAAB caused a significant increase in superoxide anion-linked ICAM-1 expression in the intima and media of the artery with increased infiltration of granulocytes in whole layers of the artery at 24 hours postapplication. A large mobilization of ED1-positive macrophages recruited to the vessel walls and surrounding blood clots from 3 days until 7 to 12 days. These variables were significantly attenuated by pretreatment with orally administered rebamipide (100 and $300 \mathrm{mg} \mathrm{kg}^{-1}$ day $\left.^{-1}\right)$.

The rat FA differs from cerebral artery in aspects such as endothelial permeability, response to vasoactive agents, and components of blood vessel matrix. ${ }^{18}$ Some similarities have, nevertheless, been identified. The time course of chronic narrowing of the FA is analogous to that observed in cerebral vasospasm after SAH, and the pathophysiology appears to be closely related with immunological and inflammatory responses. ${ }^{5,10,21,23,24}$

In the evolution of arterial narrowing and morphological changes observed in a rat FA model and of vasospasm demonstrated in human SAH, the importance of the volume and duration of perivascular clots has been emphasized. ${ }^{16,18,33}$ With regard to this aspect, several advantages of the rat FA spasm model are: 1) high survival rate; 2) an exact matched control (contralateral side); 3) easy approach for therapeutic intervention; ${ }^{18}$ and 4) technically simple and accurately controlled manipulation (for example, amount of blood, duration of bleeding). The silicone tubing technique used in this experiment allowed an appropriate amount of perivascular clot and a sufficient exposure time to the FA for inducing the pathological changes.

The cerebral vasospasm has been reported to be reduced by local administration of SOD, suggesting that excessive generation of $\mathrm{O}_{2}$ radicals contributes to the de- velopment of vasospasm after SAH..$^{29}$ This result was further supported by the fact that SOD effectively ameliorated vasospasm after SAH in SOD transgenic mice. ${ }^{9}$ Therefore, superoxide anion received attention as it modulated the growth and inflammatory processes in the adventitia and the migration of the adventitial fibroblasts for the formation of neointima. ${ }^{26,27}$ The production of $\mathrm{O}_{2}$ free radicals and expression of NADPH oxidase proteins are primarily localized in the adventitia. ${ }^{22,31}$ Recently, Wang, et al., ${ }^{31}$ reported pharmacological and biochemical evidence of a site for superoxide anion production by demon-

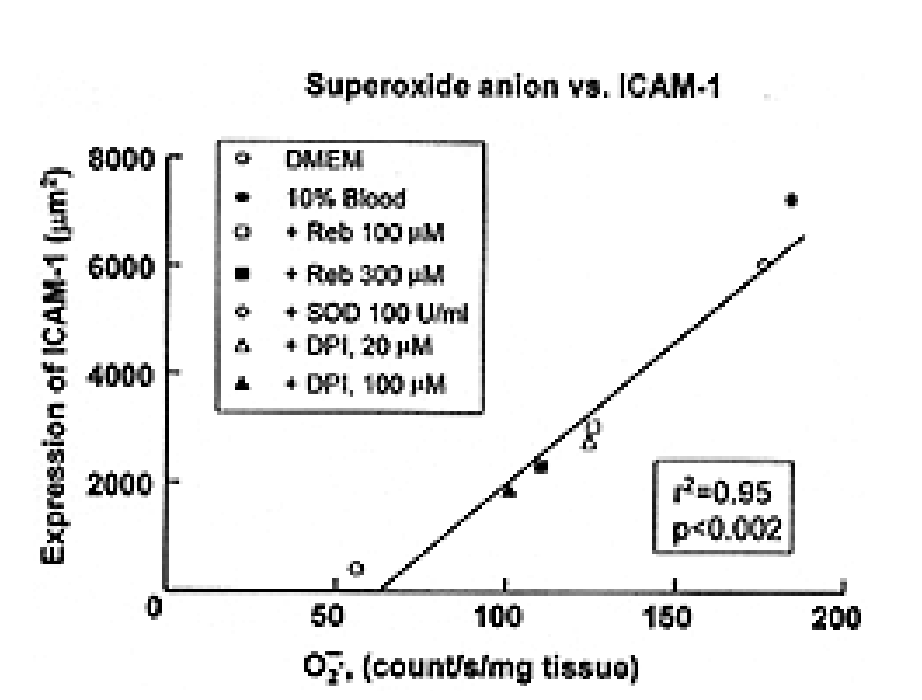

Fig. 3. Graph depicting the correlation between the generation of superoxide anion and expression of ICAM-1 in the absence (DMEM) and presence of $10 \%$ blood after pretreatment with rebamipide (Reb), SOD, and DPI. The linear regression line $\left(\mathrm{r}^{2}=\right.$ $0.95)$ showed a high significance $(p<0.002)$ between the two variables. 


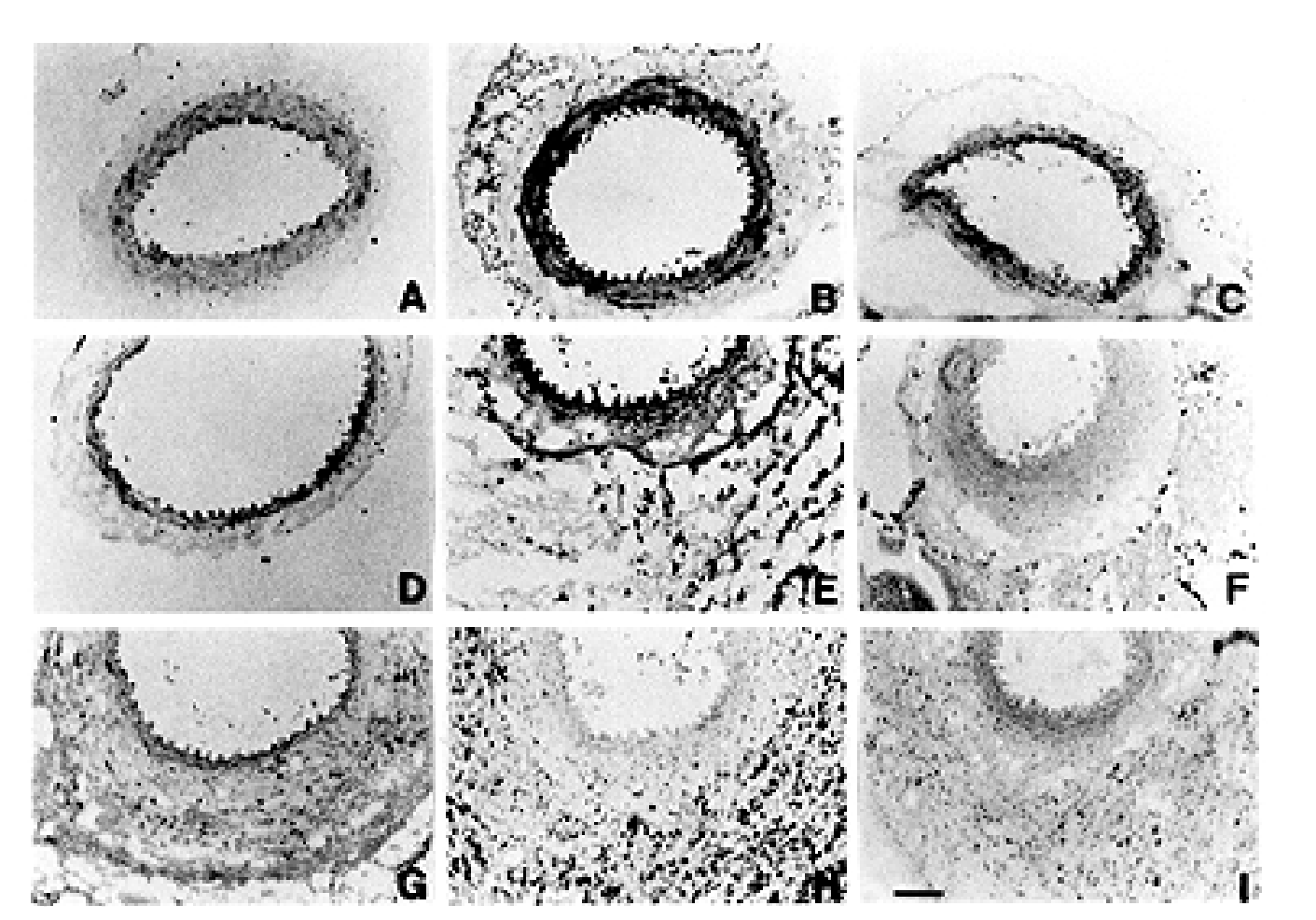

Fig. 4. Photomicrographs showing cross sections of rat FA stained for ICAM-1 (A, B, and C) and granulocytes (D, E, and $\mathrm{F})$, at 1 day posttreatment, and macrophages $(\mathrm{G}, \mathrm{H}$, and I), at 7 days posttreatment . The photomicrographs in $\mathrm{A}, \mathrm{D}$, and $\mathrm{G}$ represent control results obtained in rats treated with normal saline; the photomicrographs in $\mathrm{B}, \mathrm{E}$, and $\mathrm{H}$ represent results obtained in those treated with exposure to autologous whole blood; and the photomicrographs in C, F, and I represent results obtained in those treated with exposure to whole blood after pretreatment with rebamipide $(300 \mathrm{mg}$ $\mathrm{kg}^{-1}$ day $^{-1}$ ) from 3 days before and throughout. Note the strong immunopositive staining in the endothelium and media (B) and adventitia $(\mathrm{E}$ and $\mathrm{H})$ after application of PAAB, and minimal immunostaining of the arteries after treatment with rebamipide $(\mathrm{C}, \mathrm{F}$, and I). Immunohistochemical stain with hematoxylin counterstain, orignal magnification $\times 100$ (bar $=100 \mu \mathrm{m})$.

strating that the NADPH oxidase is localized almost exclusively in the adventitia. Consistent with these reports, analysis of our results indicated that superoxide anion production, by application blood to the aortic segments in the presence of NADPH and NADH, was significantly increased. Thus, it is suggested that there is an active ingredient of the blood-activated membrane-bound NADH/ NADPH oxidoreductase because the generation of superoxide was inhibited by diphenyleneiodonium, an inhibitor of NADH/NADPH oxidase. However, the exogenous SOD was without effect, suggesting the of difficulty of permeating the cells.

The effect of anti-ICAM-1 monoclonal antibody on inhibiting vasospasm has been demonstrated in the rat FA model. ${ }^{20}$ The ICAM-1 that remains at low levels in normal endothelial cells ${ }^{2}$ is upregulated in association with increased oxidative stress by hypoxia/reoxygenation ${ }^{15}$ and hemoglobin/heme. ${ }^{30}$ The ICAM-1-mediated inflammatory reaction was observed in the intracranial arteries in response to both ischemic injury and SAH in animal models. ${ }^{6,19}$ More recently, increased expression of ICAM-1 in response to application of blood located around the FAs in rats has been demonstrated. ${ }^{20,28}$ Expression of ICAM-1 and ICAM-1-mediated granulocyte migration constitutes a major step in the development of vasospasm after $\mathrm{SAH}^{3,7}$ In agreement with these findings, a significant increase in ICAM-1 expression in the intimal and medial layers of the artery was demonstrated at 24 hours after exposure of the artery to whole blood. In accordance with ICAM-1 expression, a significantly increased infiltration of granulocytes manifested in the same time period. Thus, it is suggested that the superoxide anion-linked ICAM-1 expression in the arterial wall plays a pivotal role in the development of the pathophysiological changes after exposure to PAAB. Here, a question arises: how is ICAM1 expression increased in the endothelial and medial layers despite periadventitial application of blood?

In the present study, a large population of macrophages recognized by ED1 monoclonal antibody (a pan-macrophage/monocyte marker) was recruited to the vascular beds and area of blood clots at 7 and 12 days after application of PAAB. Jackowski, et al., ${ }^{8}$ have demonstrated that an increased number of macrophages in the subarachnoid space maximally arose at 48 hours after SAH and declined at 5 days post-SAH. It remains unexplained why the time discrepancy has occurred. Is it due to a regional difference? The population of subarachnoid macrophage arises largely by the transformation of cells of the piaarachnoid membrane, ${ }^{8}$ whereas the macrophages in the FA is recognized by ED1 monoclonal antibody.

Rebamipide was shown to inhibit mobilization and activation of neutrophils via inhibition of production of reactive $\mathrm{O}_{2}$ species from activated neutrophils ${ }^{11}$ and via inhibition of formylmethionylleucylphenylalanine binding to 
J. M. Choi, et al.

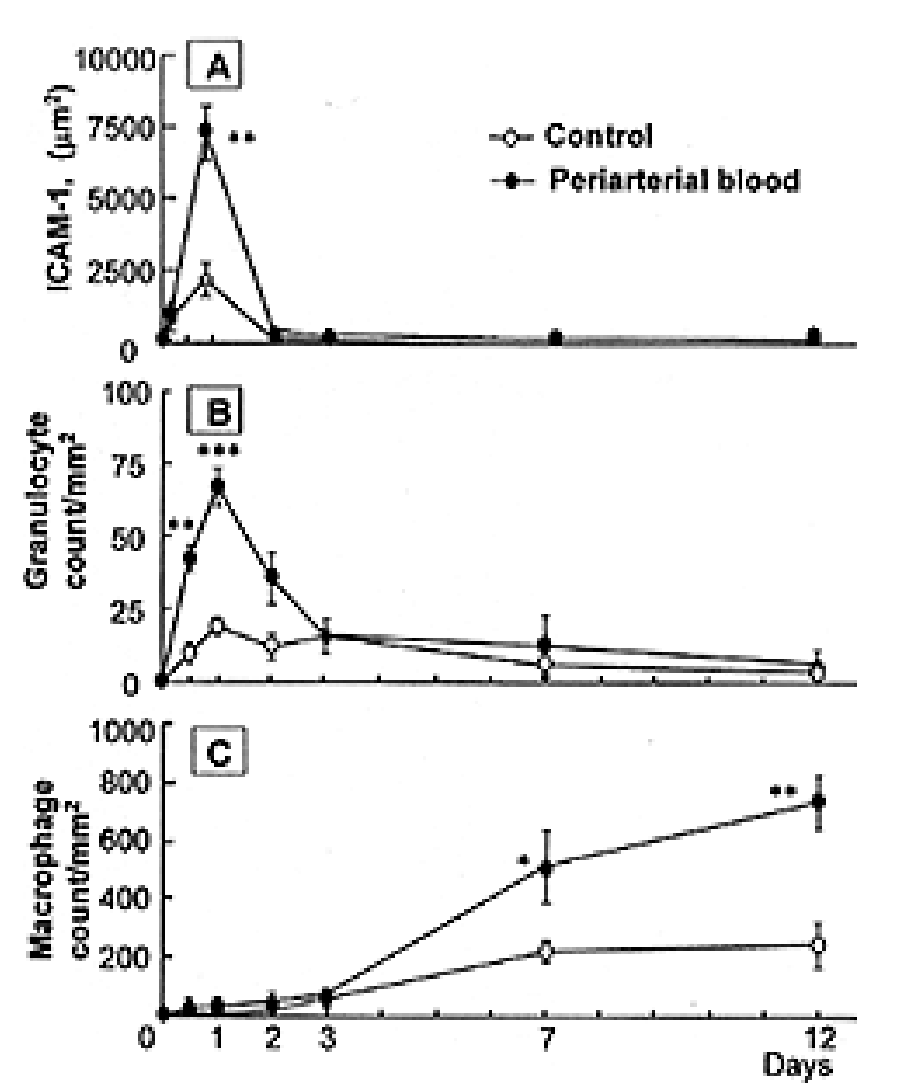

Fig. 5. Graphs depicting the time course of ICAM-1 expression (A), mobilization of granulocytes (B) detected with HIS48 monoclonal antibody, and macrophages (C) detected with ED1 monoclonal antibody in the FAs exposed to normal saline (control) and periarterial blood. An image analyzer was used to measure the intensity of ICAM-1 expression in terms of heavily immunostained area, as well as the granulocytes and macrophages in terms of counts per $\mathrm{mm}^{2}$ in area. Results are expressed as mean \pm standard error of the mean based on 4 to 6 experiments. *p $<0.05$; **p $<$ $0.01 ; * * * \mathrm{p}<0.001$ compared with the corresponding value of control group. All values are compared with the control group.

its receptors of neutrophils. ${ }^{12}$ Recently, it has been additionally demonstrated that rebamipide suppressed the expression of CD11b on neutrophils and production of interleukin-8 from gastric epithelial cells, as well as increased adherence of neutrophils to endothelial cells. ${ }^{13}$ That rebamipide strongly suppressed not only the increased superoxide anion production and ICAM-1 expression, but also PAAB-induced mobilization of granulocytes/macrophages, provides valuable information for the therapeutic approaches to relieving the vasospasm after SAH (Fig. 6).

Taken together, it is suggested that the periadventitial application of the autologous blood causes superoxide anion-linked expression of ICAM-1 and mobilization of granulocytes and macrophages, which were significantly inhibited by pretreatment with rebamipide. Furthermore, this experimental model of periadventitial application of the autologous blood to rat FA appears to be a simple and reliable means to screen the potential therapeutics for $\mathrm{SAH}$-induced vasospasm.
TABLE 1

In vivo effect of rebamipide on the ICAM-1 expression and mobilization of granulocytes and macrophages in FA exposed to normal saline and $P A A B$

\begin{tabular}{cccc}
\hline \hline & $\begin{array}{c}\text { ICAM-1 } \\
\left(\mu \mathrm{m}^{2}\right)\end{array}$ & $\begin{array}{c}\text { Granulocytes } \\
\left(\text { cells } / \mathrm{mm}^{2}\right)\end{array}$ & $\begin{array}{c}\text { Macrophages } \\
(\text { cells/mm² })\end{array}$ \\
\hline $\begin{array}{c}\text { control group } \\
\text { vehicle } \\
\text { rebamipide } \\
\mathrm{mg} \mathrm{kg}^{-1} \text { day }^{-1}\end{array}$ & $2180.8 \pm 929.1$ & $19.1 \pm 3.1$ & $220.3 \pm 36.3$ \\
30 & $1664.0 \pm 175.8$ & $15.1 \pm 3.9$ & $228.9 \pm 62.4$ \\
100 & $979.9 \pm 316.4$ & $16.2 \pm 3.9$ & $205.2 \pm 27.1$ \\
300 & $560.0 \pm 128.6$ & $14.4 \pm 2.3$ & $153.4 \pm 32.4$ \\
$\begin{array}{c}\text { PAB group } \\
\text { vehicle }\end{array}$ & $8308.5 \pm 1154.3$ & $66.9 \pm 6.3$ & $511.8 \pm 123.8$ \\
rebamipide & & & \\
30 & $4139.7 \pm 1780.6$ & $51.4 \pm 3.9$ & $558.5 \pm 55.5$ \\
100 & $1247.7 \pm 236.1 \S$ & $30.1 \pm 7.9 \ddagger$ & $447.0 \pm 83.1$ \\
300 & $977.3 \pm 285.6 \S$ & $36.8 \pm 5.1 \ddagger$ & $175.8 \pm 53.5 \dagger$ \\
\hline
\end{tabular}

* Results are expressed as means \pm standard error of the mean based on four to six experiments.

$\dagger \mathrm{p}<0.05$ as compared with vehicle.

$\ddagger \mathrm{p}<0.01$ as compared with vehicle.

$\S \mathrm{p}<0.001$ as compared with vehicle.

\section{Acknowledgment}

The authors thank Dr. Dai Hyun Yu for his critical review of the manuscript.

\section{References}

1. Asano T, Sasaki T, Koide T, et al: Experimental evaluation of the beneficial effect of an antioxidant on cerebral vasospasm. Neurol Res 6:49-53, 1984

2. Bevilacqua MP: Endothelial-leukocyte adhesion molecules. Annu Rev Immunol 11:767-804, 1993

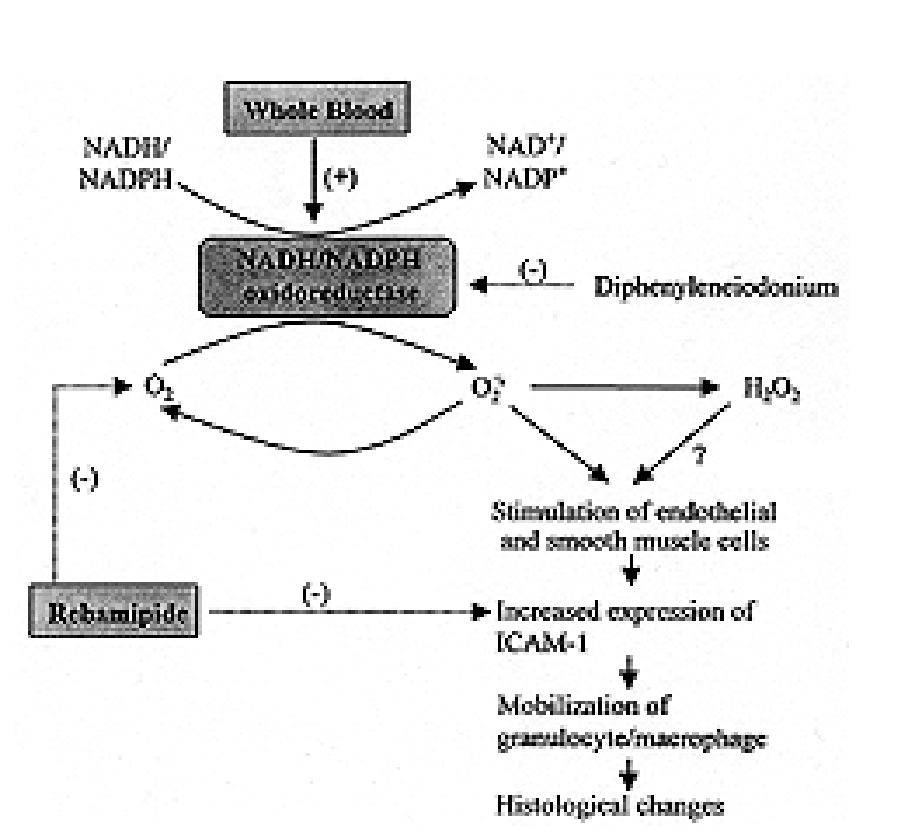

Fig. 6. Schematic drawing showing hypothesized model for superoxide anion-linked expression of ICAM-1 and mobilization of granulocytes/macrophages. 
3. Boyd AW, Wawryk SO, Burns GF, et al: Intercellular adhesion molecule 1 (ICAM-1) has a central role in cell-cell contact-mediated immune mechanisms. Proc Natl Acad Sci USA 85: 3095-3099, 1988

4. Fisher CM, Kistler JP, Davis JM: Relation of cerebral vasospasm to subarachnoid hemorrhage visualized by computerized tomographic scanning. Neurosurgery 6:1-9, 1980

5. Handa Y, Kabuto M, Kobayashi H, et al: The correlation between immunological reaction in the arterial wall and the time course of the development of cerebral vasospasm in a primate model. Neurosurgery 28:542-549, 1991

6. Handa Y, Kubota T, Kaneko M, et al: Expression of intercellular adhesion molecule 1 (ICAM-1) on the cerebral artery following subarachnoid haemorrhage in rats. Acta Neurochir 132:92-97, 1995

7. Harlan JM: Leukocyte-endothelial interactions. Blood 65: 513-525, 1985

8. Jackowski A, Crockard A, Burnstock G, et al: The time course of intracranial pathophysiological changes following experimental subarachnoid haemorrhage in the rat. J Cereb Blood Flow Metab 10:835-849, 1990

9. Kami H, Kato I, Kinouchi H, et al: Amelioration of vasospasm after subarachnoid hemorrhage in SOD-1 transgenic mice. J Soc Neurosci 23:1380, 1997 (Abstract)

10. Kasuya H, Shimizu T: Activated complement components C3a and $\mathrm{C} 4 \mathrm{a}$ in cerebrospinal fluid and plasma following subarachnoid hemorrhage. J Neurosurg 71:741-746, 1989

11. Kim CD, Hong KW: Preventive effect of rebamipide on gastric lesions induced by ischemia-reperfusion in the rat. J Pharmacol Exp Ther 275:340-344, 1995

12. Kim CD, Hong KW: Preventive effect of rebamipide on gastric mucosal cell damage evoked by activation of formyl-methionyl-leucyl-phenylalanine receptors of rabbit neutrophils. J Pharmacol Exp Ther 281:478-483, 1997

13. Kim CD, Kim HH, Hong KW: Inhibitory effect of rebamipide on the neutrophil adherence stimulated by conditioned media from Helicobacter pylori-infected gastric epithelial cells. J Pharmacol Exp Ther 288:133-138, 1999

14. Kurose I, Anderson DC, Miyasaka M, et al: Molecular determinants of reperfusion-induced leukocyte adhesion and vascular protein leakage. Circ Res 74:336-343, 1994

15. Mataki H, Inagaki T, Yokoyama M, et al: ICAM-1 expression and cellular injury in cultured endothelial cells under hypoxia/reoxygenation. Kobe J Med Sci 40:49-63, 1994

16. Mayberg MR, Okada T, Bark DH: The role of hemoglobin in arterial narrowing after subarachnoid hemorrhage. J Neurosurg 72:634-640, 1990

17. Misra HP, Fridovich I: The generation of superoxide radical during the autoxidation of hemoglobin. J Biol Chem 247: 6960-6962, 1972

18. Okada T, Harada T, Bark DH, et al: A rat femoral artery model for vasospasm. Neurosurgery 27:349-356, 1990

19. Okada Y, Copeland BR, Mori E, et al: P-selectin and intercellular adhesion molecule-1 expression after focal brain ischemia and reperfusion. Stroke 25:202-211, 1994

20. Oshiro EM, Hoffman PA, Dietsch GN, et al: Inhibition of ex- perimental vasospasm with anti-intercellular adhesion molecule-1 monoclonal antibody in rats. Stroke 28:2031-2037, 1997

21. Østergaard JR, Kristensen BØ, Svehag SE, et al: Immune complexes and complement activation following rupture of intracranial saccular aneurysms. J Neurosurg 66:891-897, 1987

22. Pagano PJ, Ito $Y$, Tornheim K, et al: An NADPH oxidase superoxide-generating system in the rabbit aorta. Am J Physiol 268: H2274-H2280, 1995

23. Pellettieri L, Carlson CA, Lindholm L: Is the vasospasm following subarachnoidal hemorrhage an immunoreactive disease? Experientia 37:1170-1171, 1981

24. Peterson JW, Kwun BD, Hackett JD, et al: The role of inflammation in experimental cerebral vasospasm. J Neurosurg 72: 767-774, 1990

25. Sakaki S, Ohta S, Nakamura H, et al: Free radical reaction and biological defense mechanism in the pathogenesis of prolonged vasospasm in experimental subarachnoid hemorrhage. J Cereb Blood Flow Metab 8:1-8, 1988

26. Scott NA, Cipolla GD, Ross CE, et al: Identification of a potential role for the adventitia in vascular lesion formation after balloon overstretch injury of porcine coronary arteries. Circulation 93:2178-2187, 1996

27. Shi Y, O'Brien JE, Fard A, et al: Adventitial myofibroblasts contribute to neointimal formation in injured porcine coronary arteries. Circulation 94:1655-1664, 1996

28. Sills AK Jr, Clatterbuck RE, Thompson RC, et al: Endothelial cell expression of intercellular adhesion molecule 1 in experimental posthemorrhagic vasospasm. Neurosurgery 41: 453-460, 1997

29. Steele JA; Stockbridge N, Maljkovic G, et al: Free radicals mediate actions of oxyhemoglobin on cerebrovascular smooth muscle cells. Circ Res 68:416-423, 1991

30. Wagener, Feldman E, de Witte T, et al: Heme induces the expression of adhesion molecules ICAM-1, VCAM-1, and E selectin in vascular endothelial cells. Proc Soc Exp Biol Med 216:456-463, 1997

31. Wang HD, Pagano PJ, Du Y, et al: Superoxide anion from the adventitia of the rat thoracic aorta inactivates nitric oxide. Circ Res 82:810-818, 1998

32. Weir B, Erasmo R, Miller J, et al: Vasospasm in response to repeated subarachnoid hemorrhages in the monkey. J Neurosurg 33:395-406, 1970

33. Zabramski JM, Spetzler RF, Bonstelle C: Chronic cerebral vasospasm: effect of volume and timing of hemorrhage in a canine model. Neurosurgery 18:1-6, 1986

Manuscript received March 20, 2000.

Accepted in final form April 25, 2000.

The authors received financial support of the Korea Science \& Engineering Foundation (K.W.H.), Korea Research Foundation, and research funds from Korea Otsuka Pharmaceutical Co., Ltd.

Address reprint requests to: Ki Whan Hong, M.D. Department of Pharmacology, College of Medicine, Pusan National University, Ami-Dong 1-Ga, Seo-Gu, Pusan 602-739, Korea. email: kwhong@hyowon.pusan.ac.kr. 